\title{
The Suture-Pull as a Refinement of the Gasket Implant Technique for Reconstruction after Endoscopic Skull Base Surgery
}

\author{
Karim Elayoubi ${ }^{1}$, Alexander G. Weil ${ }^{1}$, Ioannis Nikolaidis ${ }^{1}$, Robert Moumdjian ${ }^{1}$, Martin Desrosiers ${ }^{2}$ \\ ${ }^{1}$ Divisions of Neurosurgery, University of Montreal, Montreal, Canada \\ ${ }^{2}$ Divisions of Otorhinolaryngology, University of Montreal, Montreal, Canada \\ Email: desrosiers_martin@hotmail.com
}

Received September 1, 2012; revised October 5, 2012; accepted October 28, 2012

\begin{abstract}
Introduction: Adequate reconstruction of the skull base is the key to avoiding cerebrospinal fluid (CSF) leak following endonasal skull base surgery. The use of an endocranial "gasket" plug has been reported for this and is used in our institution. We present a simple refinement of the "gasket" technique using commonly available materials that helps ensure proper size and positioning of the gasket by applying stress on a suture attached on the center of the gasket implant. Materials and Methods: We report a case of massive CSF leak following endonasal transsphenoidal surgery for pituitary macroadenoma. The skull base was reconstructed in a multi-layered fashion with fascia lata and bony buttress reinforced with a vascularized nasoseptal flap. In order to avoid implant slippage from too-small size or malpositioning, we performed a "stress test" using traction applied to a suture attached to the center of the implant (Medpor ${ }^{\circledR}$ ), which allowed us to confirm intraoperatively that the buttress was positioned securely. Results: The patient did well without recurrence of CSF leak. At two-year follow-up, there has been no recurrence of CSF leak or occurrence local complications. We have not verified whether bony regrowth into the implant has occurred. Conclusion: The suture-pull refinement of the gasket implant technique is a simple, inexpensive and low risk method to assure secure endocranial positioning over the skull base defect, and may prevent CSF leak resulting from too-small sizing or buttress malpositioning.
\end{abstract}

Keywords: CSF Leak, Nasoseptal Flap, Skull Base Closure, Transsphenoidal Surgery

\section{Introduction}

Over the last decade, indications for endonasal endoscopic microsurgery have increased for a variety of pathologies of the anterior skull base [1]. However, the communication established between the nasal cavity and cranial cavity must be closed to avoid the development of a postoperative CSF leak. Postoperative CSF leak represents a potentially life-threatening complication, as it may be associated with pneumocephalus, meningitis, brain abscess, post-operative hydrocephalus and death [2]. Cerebrospinal fluid (CSF) leak has been reported to occur in $0.5 \%$ to $5 \%$ of cases following trans-sphenoidal surgery (TSS) for pituitary adenomas resection [3-9], $90 \%$ of which manifest as aqueous rhinorrhea [10]. Establishment of a watertight closure in skull base surgery is thus essential. An adequate reconstruction of the skull base represents the key step to decrease the risk of postoperative CSF leak [11]. Different closure techniques have been proposed based on risk factors, such as location and size of bony defect and presence of intraoperative CSF leak. While some authors suggest that hemo- static material and fibrin glue may be adequate [12], most authors recommend either multi-layered inlay-underlay grafting $[11,13]$ in addition to onlay bony buttress or pedicled vascularized nasoseptal flap (NSF) [14]. The use of a pedicled NSF, both for simple transsellar defect and for extended endonasal approach, is considered by many authors as a key step in anterior skull base reconstruction [14]. It has been shown to reduce postoperative CSF leak following extended endocranial resections to less than to 5\% [14]. Low complication rate is associated with the use of NSF; however, development of a mucocele underneath the septal flap due to persistent nasal glandular secretion has been reported [15]. Additionally, it may not be available if tumor resection has compromised vascular supply or septal architecture. Multilayer reconstruction with onlay placement of a bony buttress has been shown to prevent postoperative CSF leak, with a rate of leak similar to reconstruction with NSF [13]. However, the onlay placement of a bony buttress can be difficult when the size of the sellar defect extends to the carotid arteries and optic nerves because no bony edges 
are available for placement of bony implant [14]. For this reason, the use of a NSF may be warranted in this situation.

An endocranial "gasket" implant plug is used in combination with multi-layered free-tissue grafting and NSF in our institution. Ensuring an appropriate size and placing the implant represents technical challenges that may contribute to failure of closure and development of CSF leak with consequent morbidity. We present a simple and rapid refinement of the "gasket" technique using inexpensive, commonly available materials that helps ensure proper size and positioning of the gasket by applying stress on a suture attached on the center of the gasket implant.

Materials and methods: A 27-year-old woman presented with mild headache and gradual left homonymous hemianopsia for the last three months. A contrast-enhanced Magnetic Resonance Imaging (MRI) demonstrated a large gadolinium-enhancing lesion of the sellar and supra-sellar region, consistent with a pituitary macroadenoma (Figure 1). Endocrinological workup was normal. The patient underwent endoscopic endonasal transphenoidal approach for resection of the tumor.

\section{Operative Technique}

An incision was made in the left nasal mucosa, approximately 3 - $4 \mathrm{~mm}$ behind the mucosal-cutaneous jonction. A submucoperichondrial plan was developed, and the submucosal dissection, witch extends onto the nasal floor, was completed on the left side. A posterior ethmoidectomy was performed followed by creation of a pediculated NSF on the spheno-palatine artery. The flap was lifted and tipped into the nasopharynx until the closure. The superior choana was resected and the ostium of the sphenoidal sinus was then identified. The surgery was performed identically on the controlateral side, except for the creation of the NSF. The anterior face of the sphenoid and the intersinus septum were resected. The carotid processes were visualised and the position was confirmed with the neuronavigation guidance. The sellar bone was

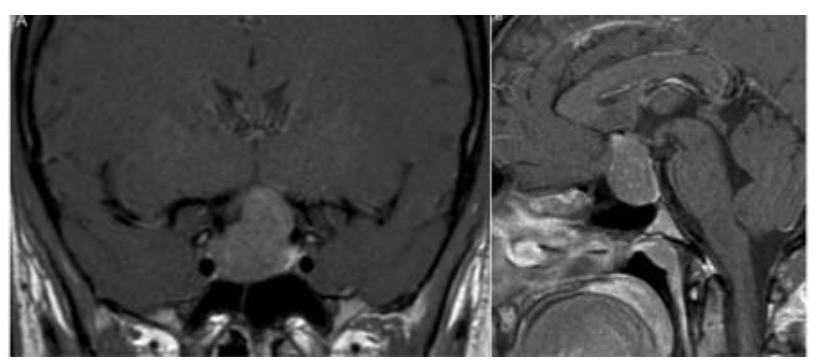

(a)

(b)

Figure 1. Coronal (a) and Sagittal (b) MRI of the sellaturcica revealing a Hardy-Vezina grade II B pituitaryMacroadenoma [18]. then cleared with the Kerrison rongeur and the dura mater was opened. Thereafter, the tumor was excised using standard microsurgical techniques. A low-flow CSF leak was noted during the intervention.

\section{Initial Closure Technique}

Autologous inlay fat graft was retrieved from the right thigh and was placed in the tumor resection cavity. A wide piece of fascia lata was layered circumferentially as an underlay between the fat graft and bony skull base margins. Thereafter, a piece of onlay Medpor ${ }^{\circledR}$ (Stryker, Hamilton, ON) was used to buttress the inlay-underlay graft in place above the sellar floor. The entire wound was covered with Tisseel ${ }^{\circledR}$ fibrin sealant (Baxter, Westlake Village, CA). Afterward, the pedicled NSF was re-flected from the nasopharynx to cover the region of the reconstruction. This flap was fixed with Tisseel ${ }^{\circledR}$. Autologous fat and fascia lata were then placed over it. Intraoperatively, there were no obvious signs of reconstruction instability. Pathology confirmed it to be a non-secreting pituitary macroadenoma.

\section{Results}

\subsection{Postoperative Course}

On the fourth postoperative day, the patient presented an important CSF leak. The leak persisted despite lumbar drainage, bed rest, and head of bed elevation.

\subsection{Modified Closure Technique}

Upon revision surgery, the fat graft and NSF were well-positioned in the sphenoid cavity. The NSF was viable without any signs of necrosis. The sphenoid cavity was penetrated and we observed, at the area of the sellar floor defect, that the Medpor ${ }^{\circledR}$ (and fascia) used to compensate the skull base defect was displaced. We concluded that this was possibly in part responsible for the CSF leak. Based on this observation, we developed an adjustment to the above-mentioned reconstruction technique, which essentially involves the attachment of a suture to the center of the Medpor ${ }^{\circledR}$ buttress which is pulled down to a secured horizontal position, buttressed against the sellar skull base defect (Figures 2 and 3). The reconstruction was completed by replacing the NSF under the bony buttress followed by Tisseel, fascia lata, and fat graft in the sphenoid cavity (Figure 3). Postoperatively, there was no recurrent CSF leak in this patient. The patient went on to make an uneventful recovery, with a postoperative CT-Scan showing a subtotal excision of the tumour (Figure 4).

\section{Discussion}

CSF leak is a common and serious complication following 
endonasal transphenoidal surgery [1]. An adequate reconstruction of the skull base represents the key step to decrease the risk of postoperative CSF leak [11]. Major risk factors for postoperative CSF leak following endoscopic pituitary surgery include size of the bony defect, and presence and importance of intraoperative CSF leak[11,13,14]. High-flow CSF leak, from opening the arachnoid cisterns or ventricles during surgery, has been

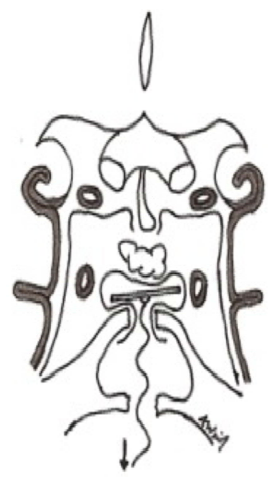

(a)

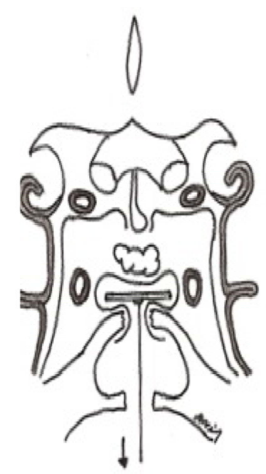

(b)

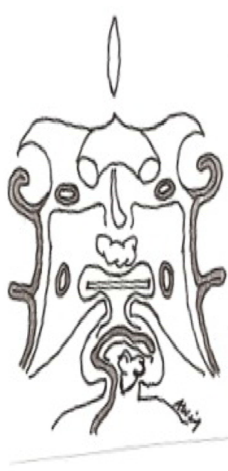

(c)
Figure 2. Illustration demonstrating the multi-layered closure for sellar reconstruction with fat graft, underlay fascia lata, and onlay Medpor ${ }^{\circledR}$ over bony defect. (a) A suture is attached on the middle of the Medpor ${ }^{\circledR}$ and (b) traction is applied to secure the medpor ${ }^{\circledR}$ horizontally on the bony defect. (c) Under the bony buttress, multi-layered reconstruction was completed with NSF, Tisseel, and fascia lata.

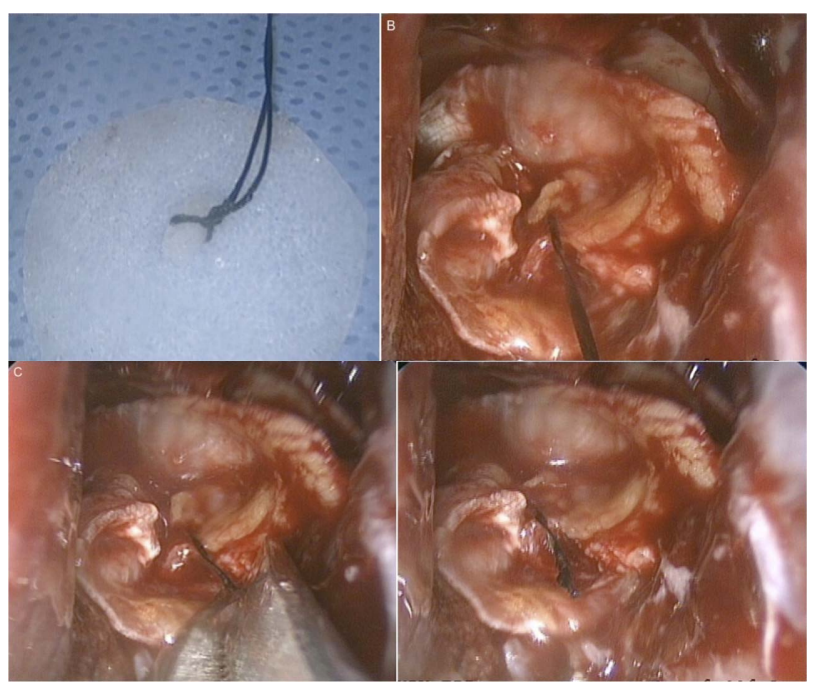

Figure 3. Intraoperative views through the endoscope showing the suture-pull technique for gasket closure. (a) A 4-0 silk suture is fixed to the center of the Medpor ${ }^{\circledR}$ bony implant; (b) Once the bony implant is placed between the fascia lata and the bony defect, traction is applied to the suture. This pulls the implant down to a horizontal position, buttressed against the sellar floor defect. It affords confirmation that the implant is well secured in place; (c) The suture is then cut with microscissors; (d) Final view of the sellar floor reconstruction.

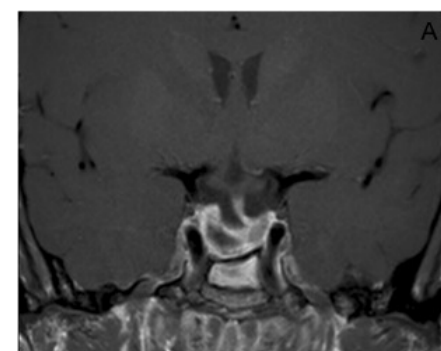

(a)

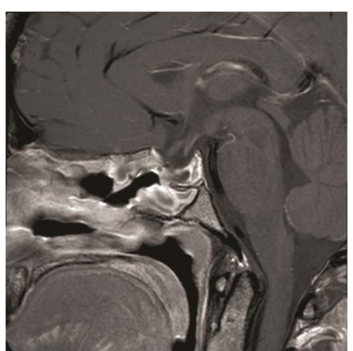

(b)
Figure 4. Postoperative coronal (a) and sagittal (b) MRI of the sella turcica at 8 weeks follow-up showing subtotal removal of the tumor.

shown to be the strongest predictor of postoperative CSF leak [14].

There is no consensus regarding the optimal closure technique following endoscopic endonasal TSS and a variety of sellar floor reconstruction methods have been recommended based on bony defect location, size and intraoperative CSF leak [11,14-16]. In cases of small defect without high-flow CSF leak, reconstruction has been performed with a variety of different techniques with a high degree of success [16]. Some authors suggest that hemostatic material and fibrin glue without grafting is adequate in most cases, even in the presence of intraoperative CSF leak [12]. However, for cases with larger bony sellar defects, especially in the presence of highflow CSF leak, some authors recommend multilayered inlay-onlay free tissue grafting $[11,13]$ with an inlay autologous fat graft in the tumor cavity, underlay fascia lata, onlay placement of bony buttress, and application of tissue sealant. However, when the size of the sellar defect extends to the carotid arteries and optic nerves, no bony edges are available to receive the onlay bony plate and reconstruction with a bony buttress is not always feasible [14]. In these cases, pediculated vascularised NSF in addition to inlay-underlay grafting is warranted and has been shown to reduce the incidence of postoperative CSF leak [14]. While many authors now recommend NSF without bony reconstruction [14], others still advocate multi-layered free tissue reconstruction with bony "gasket-seal" buttress [11,17]. Both techniques have reduces the rate of CSF leak to approximately 5\% [11,14]. In all cases with significant bony defect and/or intraoperative CSF leak, we have adopted a combined approach using multi-layered inlay fat, underlay fascia and onlay bony reconstruction (using Medpor ${ }^{\circledR}$ as graft material to close the skull base defect) in combination with NSF reconstruction.

Using this technique, we report a case of postoperative high-flow CSF leak possibly exacerbated by intraoperative malpositioning of the Medpor ${ }^{\circledR}$ buttress. The malpositioning of the Medpor ${ }^{\circledR}$ buttress, either by inadequate size or oblique orientation, may have contributed to 
the occurrence of CSF leak in this patient. Up to 5\% of patients develop CSF leak despite multi-layered reconstruction [14], and inadvertent malpositioning of the bony buttress may be an under-recognized source for CSF egress. The presence of multiple biological materials used in multi-layered reconstruction, as in this case, often obscures the view of the cartilage or heterotopic material used to close the skull base defect. For this reason, it is impossible to have a good visual confirmation of the exact spatial orientation of the bony implant. To avoid this pitfall, we developed a simple, inexpensive technique which allowed us to ensure adequate size and horizontal placement of the bony buttress against the skull base floor. This technique consists of a mechanical pulling on a stitch attached to the centre of a rigid implant (e.g. Medpor ${ }^{\circledR}$ ). This so-called stress test may help avoid slippage of the Medpor ${ }^{\circledR}$ buttress against the skull base floor from too small size or malpositioning. Following instauration of this new technique in the illustrative case, there was no recurrence of CSF leak.

The precise mechanism by which NSF and multi-layer technique exert their beneficial effects in closure of CSF leaks following endoscopic skull base surgery remains currently unknown, however possible mechanism. We believe that the 'gasket', by virtue of its of endocranial positioning and sizing larger than the bony defect, may offer a good resistance to intracranial pressure changes. However, it is probable that close approximation of the "gasket" to the adjacent skull base structures is key to success. Given that the position of the Medpor ${ }^{\circledR}$ implant is frequently concealed by both its endocranial position and the fascia used for reconstruction, positioning errors may occur leading to potential slippage and failure. Performing a "stress-test" on the implant by exerting a traction on a stitch attached to the centre of the rigid implant may help to ensure the correct positioning of the implant over the skull base defect by ensuring tight appositioning to adjacent structures and that displacement secondary to too-small size be detected early.

In the patient described in this report, even if the only obvious peri-operative sign of reconstruction instability was the implant displacement, we could not completely exclude a malpositioning of the NSF as the cause of CSF egress. We are also aware that there is actually no evidence supporting the fact that the addition of an implant buttress to the NSF decreases CSF leak following skull base reconstruction. However, many surgeons still incorporate an implant buttress when performing a skull base reconstruction following endonasal endoscopic skull base surgery. Our refinement of this standard gasket implant buttress technique is a simple, safe, and inexpensive "stress test" for the implant buttress to provide the surgeon immediate feedback and assurance of appropriate gasket size and positioning against the skull base de- fect. As persistent CSF leak represents a serious clinical complication and is still reported to occur in approximately $5 \%$ of endonasal transsphenoidal surgery in both NSF and bony graft reconstruction techniques, there is still place for improvement. We propose the suture-pull gasket implant technique as a potential refinement that may help to decrease the rate of CSF leak. Further studies are needed in order to confirm the incidence of bony implant malpositioning, its impact on postoperative CSF leak, and the efficacy related to suture-pull modified gasket technique.

\section{Conclusion}

Multi-layered reconstruction with fat graft, fascia lata, and cartilage, bone or synthetic gasket closure has been described for the prevention of CSF leak following endoscopic pituitary surgery. Failure of this technique may result from inadvertent orientation of the gasket, resulting in continued CSF egress. We report a new adjustment to this anterior skull base closure technique in which a suture centered on the gasket (medpor ${ }^{\circledR}$ ) is used to pull it in a secured horizontal position. This technique, both easy and safe to perform, may minimize the risk of massive postoperative CSF leak in some cases.

\section{REFERENCES}

[1] C. D. Gandhi, L. D. Christiano, J. A. Eloy, C. J. Prestigiacomo and K. D. Post, "The Historical Evolution of Transsphenoidal Surgery: Facilitation by Technological Advances,” Neurosurg Focus, Vol. 27, No. 3, 2009, p. E8. doi:10.3171/2009.6.FOCUS09119

[2] P. M. Black, N. T. Zervas and G. L. Candia, "Incidence and Management of Complications of Transsphenoidal Operation for Pituitary Adenomas," Neurosurgery, Vol. 20, No. 6, 1987, pp. 920-934.

[3] P. Cappabianca, L. M. Cavallo, F. Esposito, V. Valente and E. de Divitiis, "Sellar Repair in Endoscopic Endonasal Transsphenoidal Surgery: Results of 170 Cases,” Neurosurgery, Vol. 51, No. 6, 2002, pp. 1365-1372.

[4] C. Martin-Martin, G. M. Capoccione, R. S. Garcia and F. Espinosa-Restrepo, "Surgical Challenge: Endoscopic Repair of Cerebrospinal Fluid Leak,” BMC Research Notes, Vol. 5, 2012, p. 459. doi:10.1186/1756-0500-5-459

[5] I. Ciric, A. Ragin, C. Baumgartner and D. Pierce, "Complications of Transsphenoidal Surgery: Results of a National Survey, Review of the Literature, and Personal Experience,” Neurosurgery, Vol. 40, 2007, pp. 225-237. doi:10.1097/00006123-199702000-00001

[6] F. Esposito, J. R. Dusick, N. Fatemi and D. F. Kelly, "Graded Repair of Cranial Base Defects and Cerebrospinal Fluid Leaks in Transsphenoidal Surgery,” Neurosurgery, Vol. 60, No. 2, 2007, pp. 295-304. doi:10.1227/01.NEU.0000255354.64077.66

[7] D. F. Kelly, R. J. Oskouian and I. Fineman, “Collagen Sponge Repair of Small Cerebrospinal Fluid Leaks Obvi- 
ates Tissue Grafts and Cerebrospinal Fluid Diversion after Pituitary Surgery," Neurosurgery, Vol. 49, No. 4, 2001, pp. 885-890.

[8] H. Nishioka, H. Izawa, Y. Ikeda, H. Namatame, S. Fukami and J. Haraoka, "Dural Suturing for Repair of Cerebrospinal Fluid Leak in Transnasal Transspenoidal Surgery,” Acta Neurochirurgica, Vol. 151, No. 11, 2009, pp. 1427-1430.

[9] G. T. Tindall, E. J. Woodard and D. L. Barrow, "Pituitary Adenomas: General Considerations,” In: M. L. J. Apuzzo, et al., Eds., Brain Surgery: Complication Avoidance and Management, Churchill Livingstone, New York, 1993, pp. 269-276.

[10] E. Kim and P. T. Russell, "Prevention and Management of Skull Base Injury," Otolaryngologic Clinics of North America, Vol. 43, No. 4, 2010, pp. 809-816. doi:10.1016/j.otc.2010.04.018

[11] A. Tabaee, V. K. Anand, S. M. Brown, J. W. Lin and T. H. Schwartz, "Algorithm for Reconstruction after Endoscopic Pituitary and Skull Base Surgery,” Laryngoscope, Vol. 117, No. 7, 2007, pp. 1133-1137. doi:10.1097/MLG.0b013e31805c08c5

[12] L. Seda, R. B. Camara, A. Cukiert, J. A. Burratini and P. P. Mariani, "Sellar Floor Reconstruction after Transsphenoidal Surgery Using Fibrin Glue without Grafting or Implants: A Technical Note," Surgical Neurology, Vol. 66, No. 1, 2006, pp. 46-49. doi:10.1016/j.surneu.2005.10.021

[13] G. Zielinski, J. K. Podgorski, A. Koziarski and Z. Potakiewicz, "Reconstruction of the Sellar Floor in Transsphenoidal Surgery: Our Experience in $818 \mathrm{~Pa}-$ tients,” Neurologia I Neuroriurgia Polska, Vol. 40, No. 4, 2006, pp. 302-311.

[14] M. R. Patel, M. E. Stadler, C. H. Snyderman, R. L. Carrau, A. B. Kassam, A. V. Germanwala, et al., "How to Choose? Endoscopic Skull Base Reconstructive Options and Limitations,” Skull Base, Vol. 20, No. 6, 2010, pp. 397-493. doi:10.1055/s-0030-1253573

[15] R. Vaezeabshar, P. H. Hawang, G. Harsh and J. H. Turner, "Mucocele Formation under Pediculated Nasoseptal Flap," American Journal of Otolaryngology, Vol. 33, No. 5, 2012, pp. 634-636. doi:10.1016/j.amjoto.2012.05.003

[16] H. M. Hegazy, R. L. Carrau, C. H. Snyderman, A. Kassam and J. Zweig, "Transnasal Endoscopic Repair of Cerebrospinal Fluid Rhinorrhea: A Meta-Analysis," Laryngoscope, Vol. 110, No. 7, 2000, pp. 1166-1172. doi:10.1097/00005537-200007000-00019

[17] L. Z. Leng, S. Brown, V. K. Anand and T. H. Schwartz, "'Gasket-Seal' Watertight Closure in Minimal-Access Endoscopic Cranial Base Surgery,” Neurosurgery, Vol. 62, Suppl. 2, 2008, Discussion ONSE343.

[18] J. L. Vezina, J. Hardy and M. Yamashita, "Microadenomas and Hypersecreting Pituitary Adenomas," Arq Neuropsiquiatr, Vol. 33, No. 2, 1975, pp. 119-127. 